\title{
Application of Artificial Intelligence (AI) Tools in Integrated Pest Management (IPM) - An Insect - Plant Interaction Perspective
}

\section{S Prem Mathi Maran*}

Director, G.S. Gill Research Institute, Guru Nanak College, Guru Nanak Salai, Velachery, Chennai, India

*Corresponding Author: S Prem Mathi Maran, Director, G.S. Gill Research Institute, Guru Nanak College, Guru Nanak Salai, Velachery, Chennai, India.
Received: November 30, 2021

Published: December 10, 2021

(C) All rights are reserved by $\mathbf{S}$ Prem Mathi

Maran.

\begin{abstract}
Integrated Pest Management (IPM), is a safe and eco friendly pest control strategy, which is in practice for the past three decades. Utilization of semiochemicals and insect-plant interaction processes in IPM is a topic of research now a day. In this current concept report, which is based on the available evidences that uses the semiochemicals for IPM, the author explains the potential use of sensors, artificial intelligence (AI) and Machine Learning (ML) procedures, which can be integrated in the modern agriculture for IPM measures. A workable model of AI and ML is explained in Push-Pull strategy of IPM.
\end{abstract}

Keywords: Integrated Pest Management; Semiochemicals; Infochemicals; Insect-plant Interaction; Olfactory System; Molecular Model; Biosensors; Nano Sensors; Artificial Intelligence; Machine Learning

\section{Introduction}

The scientific investigations on insect plant interactions is a subject of importance for more than a century and it is a frontier subject among the entomologist, agriculturist and environmentalists. The insect plant interaction is a holistic subject which includes several disciplines of science. The insect plant interaction is a molecular level mechanism which is mediated mainly by herbivore associated molecular pattern (HAMPs) [1]. The host plant releases defensive chemical molecules the moment they are attacked by herbivores insect pests. This defensive activity is primarily governed by few signaling mechanisms which include mitogen-activated kinase (MAP kinase), jasmonic acid (JA), salicylic acid(SA) and ethylene (ET) pathways. These signalling pathways regulate the gene expressions, protein synthesis and synthesis of defensive molecules. This method is referred as direct defensive method. There is an indirect method of defensive mechanism where in the plants emit volatile organic compounds (VOC), which are secondary and tertiary plant metabolites thus attracting natural enemies of the herbivorous insect pests; hence these pests are controlled naturally by their natural enemies (tritrophic interaction) [1].

Integrated pest management (IPM) is considered as a safer method of controlling herbivorous insect pests. The indiscriminate usages of chemical/synthetic pesticides for controlling these herbivorous insect pests lead to environmental imbalance and environmental threats. This leads to the formation of integrated pest management strategies. There are many IPM methods, the promising among them is usage of semiochemicals which are informative molecules that constitute tritrophic interaction (host plant-pestnatural enemies).

Semiochemicals are considered as an important tool in integrated pest management (IPM) [2]. These semiochemicals are compli- 
mentary or alternative compounds to chemical pesticides in IPM strategies. The olfactory response of any herbivorous insect pests to host plant volatiles determine the food, mating, oviposition and other insect pest behaviors. IPM of herbivorous insect pests utilize the olfactory chemical signals to monitor, mass trap, lure, kill, mate distribution and push/pull strategy. These control strategies can be used singly or in combination with one another. It is recorded, the combination of these chemical communication signals are more efficient in IPM as attraction of a herbivorous insect pests by a multiple chemical stimuli is higher than a single stimulus $[3,4]$.

Sensor based detection of semiochemicals was extensively reviewed by Brezolin., et al. (2018) [5]. Work on sensorics has contributed to the development of many tools like chemo, bio and nano sensors to detect the selective semiochemicals.

To develop AI and ML strategies in IPM using semiochemicals need a detailed study on the insect plant interactions. A better understanding on the olfactory and chemosensory systems, feeding preferences, sensilla on the antennae and mouth parts of herbivorous insect pests is imperative as these insects detects and differentiates various semiochemicals even at extremely lower concentrations and even the insects distinguish the various isomeric molecules of semiochemicals [3]. Hence a multidisciplinary research work is the need for the hour on the chemical ecology of insects to develop modern tools and to develop artificial intelligence (AI) tools and machine learning (ML) strategies for IPM. This current concept paper, which is based on various evidences, gives a detailed insight of steps, concepts and procedures of a new IPM strategy using semiochemicals and artificial intelligence methods.

\section{Classification of semiochemicals}

Semiochemicals are otherwise called as informative molecules that convey chemical messages between insect-insect and plantinsect, which evoke physiological and behavioral responses within and between species.

The following flow chart depicts the broad outline classification of semiochemicals [6].

\section{Semiochemivals in IPM}

The efficacy of semiochemicals molecule in IPM greatly depends on the physico-chemical properties and the environmental conditions. The key physico-chemical properties are molecular

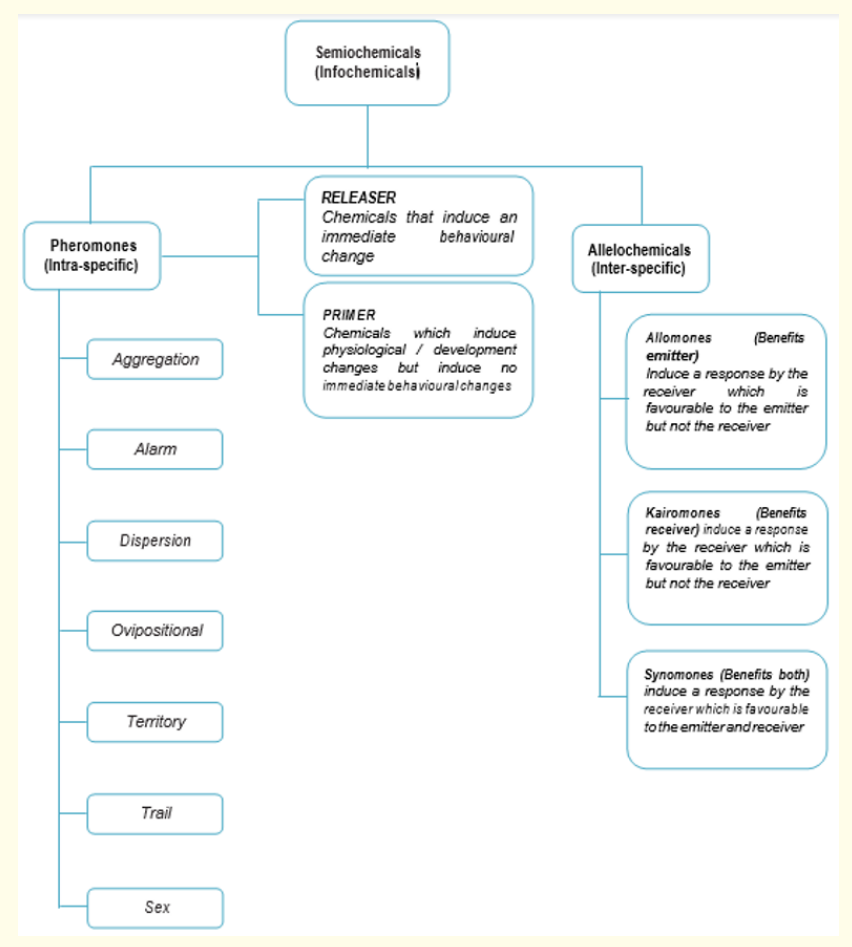

Flow Chart 1

structure, solubility and volatility in the air and it's life time in the environment. The environmental condition that determines the effectiveness of these semiochemicals is mainly the temperature which influences the stability, solubility, volatility and life time [7].

Sex pheromones of Lepidopteran pests are the first recognized and synthesized ones, which has very large scale application in IPM. As semiochemicals are not killing agents like chemical pesticides, Reddy., et al. (2005) [8], set few strategies of using semiochemicals in IPM to a) monitor the pest population b) possibilities of controlling the pests and c) altering the behavioural pattern of herbivorous insect pests and or it's natural enemies.

As these semiochemicals are non toxic, low dosages to exert better control over pests, having adverse effects only on target pests, non persistent and eco friendly and target pests cannot develop resistance to these biological molecules the usage and development of semiochemicals based products are emerging now. The application of these volatile compounds in IPM requires very low concentrations. Even at the low concentrations these semiochemi- 
cals exert good control over insect pests as it has high volatility and diffuses to long distances.

Herbivore induced plant volatiles is also one of a promising tool in IPM as it attracts the natural enemies too to find their prey.

Sampling tools to study semiochemicals used in insect plant interaction

To develop the sensors, AI and ML programmes, a thorough knowledge on the molecular structure, physico chemical properties and biological properties of a semiochemicals and VOC is imperative. To elucidate all the above properties, extraction, analysis and biological assays need to be done. The following are the various methods used for extraction, analysis and bioassays of semiochemicals and VOC's. (Cornelio., et al. 2019) [9].

Extraction of semiochemicals

Solvent Assisted Extraction (SAE) Distillation.

Trapping of semiochemicals

- $\quad$ Enclosure techniques.

- $\quad$ Static (SHS) and Dynamic Head space (DHS) sampling. Solid Phase Extraction (SPE).

- $\quad$ Solid phase micro Extraction (SPME).

- $\quad$ Head space -solid phase micro extraction (HS-SPME).

Analytical tools to identify semiochemicals

- $\quad$ Gas Chromatography (GC)

- Gas chromatography coupled to Ionization Detectors (GCFID)

- Gas chromatography coupled to Electroantennography Detectors (GC-EAD) Gas chromatography coupled to Mass Spectrometry (GC-MS)

- $\quad$ Nuclear Magnetic Resonances (NMR).

Bioassay of semiochemical recognition by insects

- $\quad$ Electroantennography (EAG)

- $\quad$ Single sensillum recording (SSR).

- Behavioural Experiments:

- $\quad$ Linear Olfactometry.
- $\quad$ Two path olfactometry.

- Multiple path olfactometry.

- Wind tunnel assay

Sensor based detection

Biological molecular recognition system (olfactory organs)

The insect olfactory system utilise highly sophisticated molecular mechanisms which involve the olfactory receptors activation which leads to a rapid detection in real time measurement systems. The sensory organs/olfactory organs of a insect include antennae, mouth parts and ovipositor (Panda, Kush 1995) [10]. The insects olfactory system is far superior in terms of sensitivity, selectivity, response time. The individual chemosensory neurons of insects have specific receptor sites. Stimulation of a olfactory system by semiochemicals produce specific binding proteins (BPs), that binds to specific receptor sites. The Lepidopteran olfactory system is classified as 4 families based on the proteins they release in response to a semiochemicals stimuli. They are Chemosensory Proteins (CSPs), Odorant Binding Proteins (OBPs), Odorant Degrading Enzymes (ODEs) and Chemosensory Receptors (CRs). The Odorant Binding proteins (OBPs or BPs) are Pheromone Binding Proteins (PBPs), General Odorant Binding Proteins (GOBPs) and Antennal Binding Protein X (ABPx). The odorant degrading enzymes are Estrases (CXEs), Aldehyde Oxidases (AOXs), Alcohol Dehydrogenases (ADs), Cytochrome P-450, Glutathione S-Transferase and UDPGlucuronosyl Transferase. The chemo sensory receptors in the insect olfactory system has Odorant receptors (ORs), Ionotropic receptors (IRs), Gustatory Receptors (GRs) and Sensory Neuron Membrane Protein (SNMPs). (Godoy., et al. 2021) [11].

The responses evoked in insects by these semiochemicals by the above mechanisms shall be attractive or repellent. The behavioral response of insects for finding an odour source is classified into 3 mechanisms.

- Chemotaxis: Wherein he insects body is directly aligned towards the odour source because of the gradient of the odour molecule.

- Orthokinesis and Klinokinesis: Wherein the insects do not recognize the odour direction, but stimulated for moving, depend upon the odour concentration or turning (klinokinesis). 
- $\quad$ Anemotaxis: The attached chemical molecule stimulate the recipient insect to fly upwind [12].

Sensors in integrated pest management

Sensors are defined as the device which can transform the outcome of a chemical or physical reaction that happens in the live organism or in the environment into a useful signal which can be analysed using specific programs.

Sensors are classified based on the principle of detection as a) physical sensor b) chemical sensor and c) biosensors. A sensor is user friendly when it is having properties like sensitivity, stability, fast response time and lower detection limits (Brezolin., et al. 2018) [5].

Chemical sensors are very effective tools for detecting even the lowest quantities of volatile organic compounds emanating from host plants, insects and in the agro ecosystem. In chemical sensors, the volatile molecule binds with the sensitive material, exert change in the sensitive material. These changes which are normally electrochemical are converted into a signal, which can be analyzed. These sensor materials are acting as a transducer. There are semiconductor sensors which can detect these volatile organic compounds in the environment from 5 to $500 \mathrm{ppm}$ with a fast response time [13-15].

Biosensors use biological components such as a whole organism, an organ, a tissue, a cell, nucleus acid, genetic material and enzymes to detect and respond to a specific volatile compound. The signals transduced which is in proportion to the concentration of the VOC in the environment by a biosensor in response to a VOC is measured optically, electrochemically, thermometrically, magnetically or micromechanically. There are biosensors that show a rapid response time of $\sim 13$ seconds (Brix., et al.) [16-18].

The flow chart 2 explains the process sequences of developing an Artificial Intelligence (AI) method based IPM strategy.

Table 1 explains the potential control methods using sensors, AI and ML integrations.

Semiochemicals play a significant role in the behavioral pattern of herbivorous insect pests in finding host plant location and acceptance for feeding and oviposition (columns 1 and 2) [19,20]. The host plant chemical cues also attract natural enemies, which balances the agro ecosystem. Based on the strength, volatility, dif-

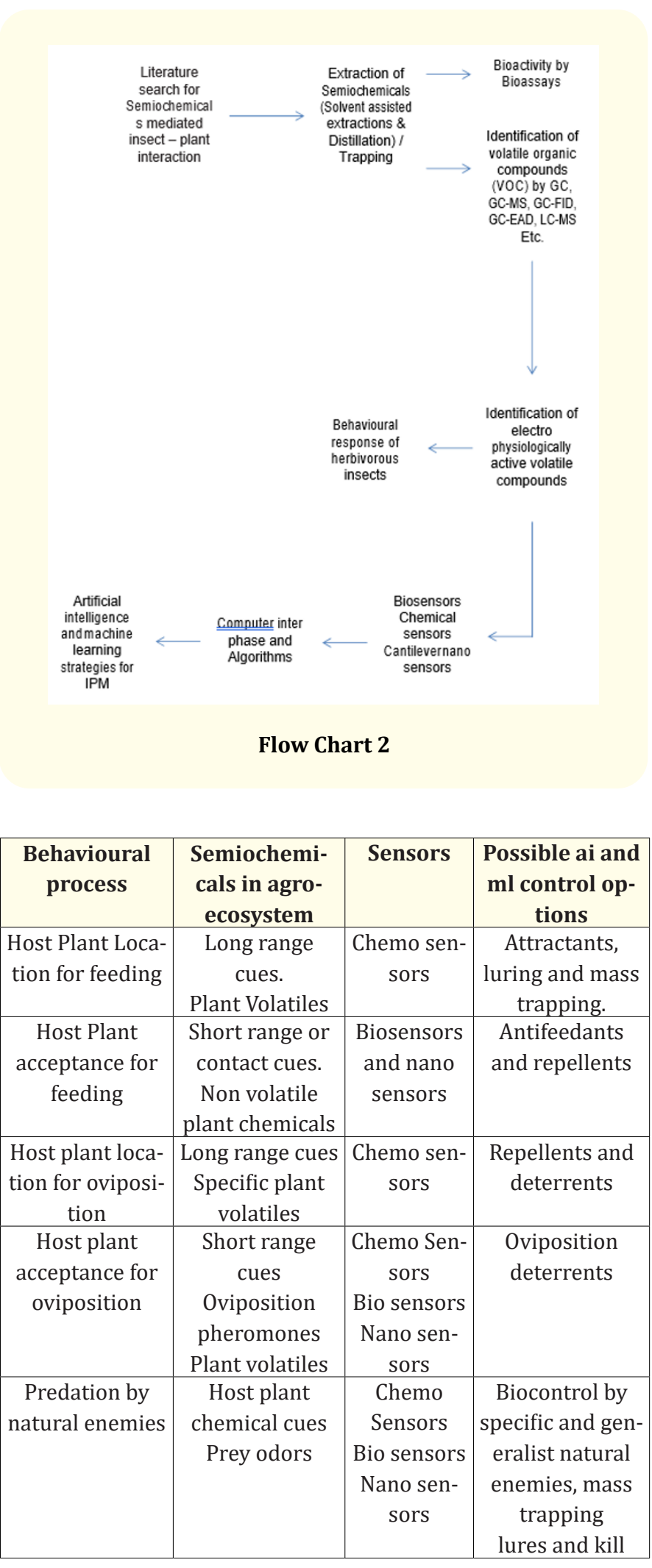

Table 1 
fusibility of the chemical cues, different types of sensors are used (column 3) [21]. These sensors transduce the chemical signals into electromagnetic signals. Based on the signals generated, the AI and ML system responds and does the control measures (column 4) using a pre programmed hardware and software systems.

\section{Push-pull strategy of IPM}

The integration of AI and ML in IPM is a noval strategy. Scientists at G.S. Gill Research Institute, Guru Nanak College, Chennai, developed a new concept of integrating the AI and ML methods in IPM, very specifically on the push-pull strategy of IPM. The pushpull strategy of IPM was discussed in detail by Cook., et al. (2007) [4]. The following flow chart explains the above concept.

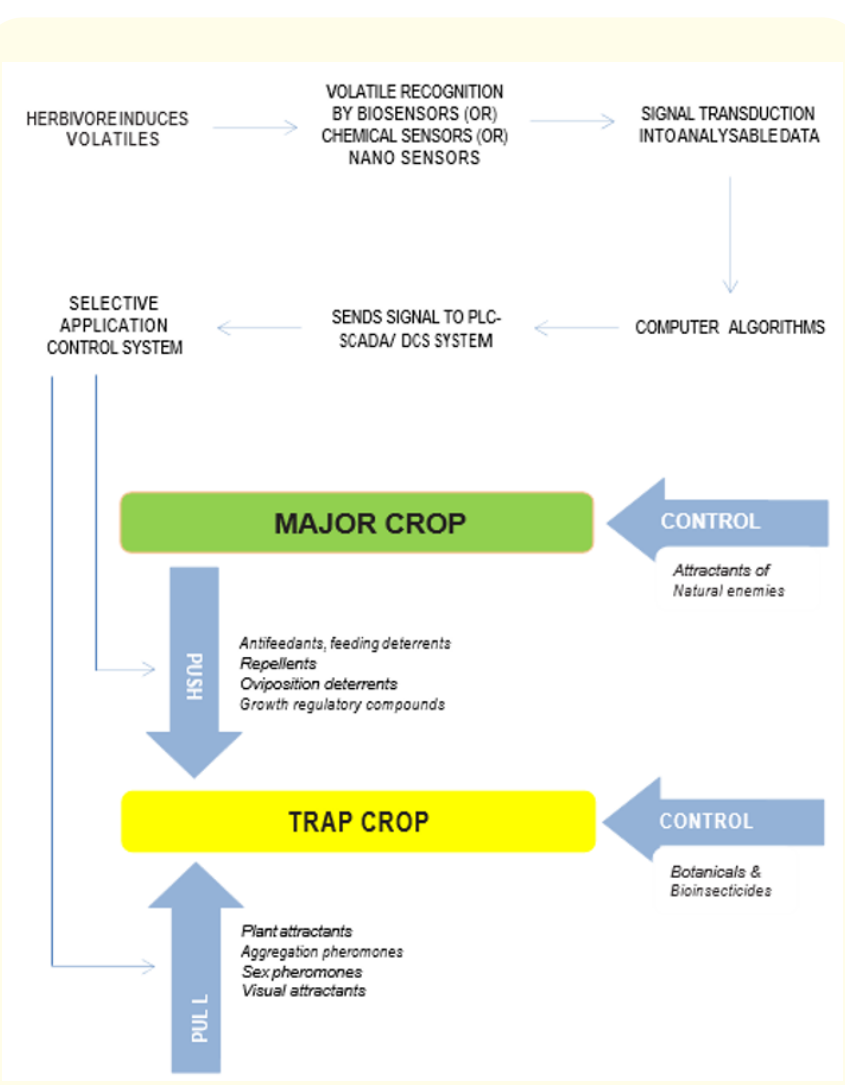

Flow Chart 3

\section{Conclusion}

Artificial Intelligence (AI) and Machine Learning (ML) are very promising tools that can be used in Integrated Pest Management (IPM) strategies. This current concept report reveals the promising possibilities and concepts based on evidence based studies. A further research on such aspects is the need for the universe as these are eco friendly, efficient and economical strategies.

\section{Acknowledgement}

The author sincerely thank Sardar Manjit Singh Nayyar, General Secretary and Correspondent, Guru Nanak Educational Society for the supports and encouragements.

\section{Bibliography}

1. Anupam Varshney Sharma and Vachaspati Mishra. "Simplified Prespective of Complex Insect-Plant Interactions". Molecular Mechanisms to Chemical Ecology (2021): 399-415.

2. Alice L Mauchline., et al. "Semiochemical-based alternatives to synthetic toxicant insecticides for pollen beetle management". Arthropod-Plant Interactions 12 (2018): 835-847.

3. Abd El-Ghany NM. "Semiochemicals for controlling insects pests". Journal of Plant Protection Research 50.1 (2019): 1-11.

4. Cook SM., et al. "The use of push-pull strategies in integrated pest management". Annual Review of Entamology 52 (2007): $375-400$.

5. Brezolin AN., et al. "Tools for detecting insect semiochemicals: a review". Analytical and Bioanalytical Chemistry 410 (2018): 4091-4108.

6. Nordlund DA., et al. "Semiochemicals". Their role in pest control, John Wiley and sons, New York (1981).

7. Tamoghna Saha and Nithya Chandran. "Chemical ecology and pest management: A review". International Journal of Chemical Studies 5.6 (2017): 618-621.

8. Reddy GVP., et al. "Development of a semiochemical-based trapping method for the New Guinea sugarcane weevil. Rhabdoscelus obscures". Journal of Applied Entomology 129 (2005): 65-69.

9. Ricardo Barbosa-Cornelio., et al. "Tools in the Investigation of Volatile Semiochemicals on Insects: From sampling to statistical analysis". Insects 10.8 (2019).

10. Panda N and Kush GS. "Host plant resistance to insect". Wallingford, UK: CAB international (1995): 448. 
11. Ricardo Goboy., et al. "An overview of antennal esterases in Lepidoptera)". Frontiers in Physiology (2021).

12. El-Shafie HAF and Faleiro JR. "Semiochemicals and their potential use in Pest Management" (2017).

13. Zhu C., et al. "Electrochemical sensors and biosensors based on nanomaterials and nanostructures". Analytical Chemistry 87.1 (2015): 230-249.

14. Lu Y., et al. "Olfactory biosensor for insect Semiochemicals analysis by impedance sensing of odorant-binding proteins on interdigitated electrodes". Biosensors and Bioelectronics 67 (2015): 662-669.

15. Brattoli M., et al. "Odour detection methods: olfactometry and chemical sensors". Sensors (2011).

16. Turner AP. "Biosensors: sense and sensibility". Chemical Society Reviews (2013).

17. Olsson SB and Hansson BS. "Electroantennogram and single sensillum recording in insect antennae". Methods in Molecular Biology 1068 (2013): 157-177.

18. Dai $\mathrm{C}$ and Choi S. "Technology and applications of microbial biosensor". Open Journal of Applied Biosensor 2.3 (2013).

19. Miller JR and Strickler KL. "Plant herbivore relationships: Finding and accepting host plants". In: Bell WJ, Carde RT, editors. Chemicals Ecology of Insects. Boston, MA: Springer (1984): 127-157.

20. Nishida R. "Sequestration of defensive substances from plants by Lepidoptera”. Annual Review of Entomology 47 (2002): 57 92.

21. Karlson $\mathrm{P}$ and Butenandt A. "Pheromones (Ectohormones) in insects". Annual Review of Entomology 4 (1959): 39-58.

\section{Assets from publication with us}

- Prompt Acknowledgement after receiving the article

- Thorough Double blinded peer review

- Rapid Publication

- Issue of Publication Certificate

- High visibility of your Published work

Website: www.actascientific.com/

Submit Article: www.actascientific.com/submission.php

Email us: editor@actascientific.com

Contact us: +919182824667

Citation: S Prem Mathi Maran. “Application of Artificial Intelligence (AI) Tools in Integrated Pest Management (IPM) - An Insect - Plant Interaction Perspective". Acta Scientific Agriculture 6.1 (2022): 11-16. 